\title{
Material Classification for Printed Circuit Boards by Spectral Imaging System
}

\author{
Abdelhameed Ibrahim, Shoji Tominaga, and Takahiko Horiuchi \\ Department of Information Science, Graduate School of Advanced Integration Science, \\ Chiba University, Japan \\ ibrahimegraduate.chiba-u.jp, \{shoji,horiuchi\}@faculty.chiba-u.jp
}

\begin{abstract}
This paper presents an approach to a reliable material classification for printed circuit boards (PCBs) by constructing a spectral imaging system. The system works in the whole spectral range [400-700nm] and the high spectral resolution. An algorithm is presented for effectively classifying the surface material on each pixel point into several elements such as substrate, metal, resist, footprint, and paint, based on the surface-spectral reflectance estimated from the spectral imaging data. The proposed approach is an incorporation of spectral reflectance estimation, spectral feature extraction, and image segmentation processes for material classification of raw PCBs. The performance of the proposed method is compared with other methods using the RGB-reflectance based algorithm, the k-means algorithm and the normalized cut algorithm. The experimental results show the superiority of our method in accuracy and computational cost.
\end{abstract}

Keywords: Spectral imaging system, material classification, printed circuit board, spectral reflectance, region segmentation, k-means, normalized cut.

\section{Introduction}

Material classification is one of the important problems in computer vision, which is depending on surface-spectral reflectance of observed materials. The surface-spectral reflectance of objects is inherent to the material composition. Therefore this inherent physical property can be helpful in recognizing objects and segment regions in the illumination invariant way. With computer hardware and camera advances, new computer vision algorithms should be developed and applied in industry. A PCB in a variety of industries is one of the most complicated objects to understand from the observed image. The surface layer of a raw PCB is composed of various elements, which are a mixture of different materials and the area of each element is very small. These features make the machine inspection difficult.

There are numerous algorithms, approaches, and techniques in the area of PCB inspection nowadays [1-5]. Most of them are based on binary or gray-scale images subtraction to classify board defects. Chang et al. [1] developed a case-based reasoning evolutionary model to classify defects of PCB images based on binary image difference. An eigenvalue-based similarity measure between two gray-level images is 
proposed in [2] with application of assembled PCB defect inspection. Ibrahim et al. [3] applied image difference operation in the wavelet-domain image in order to minimize computational time for PCB inspection. A contour-based window extraction approach for bar PCB inspection from gray-scale images is proposed in [4]. Leta et al. [5] presents a new algorithm to solve PCB inspection problem based on gray-level images subtraction technique.

Since image understanding is the first and foremost step in the inspection of PCBs, an improved image capturing system supports detection of defects. In our previous works, a material classification algorithm was proposed based on surface-spectral reflectance [6], [7]. However, due to limitations of spectral imaging, the method was too simple to obtain enough accuracy in segmentation results of PCB images. The present paper presents a non-contact measurement approach to performing a reliable material classification for PCBs that can be used in inspection system by constructing an improved spectral imaging system. The proposed approach is an incorporation of spectral reflectance estimation, spectral feature extraction, and image segmentation processes for material classification of raw PCBs.

The performance of our spectral image segmentation algorithm is compared with typical segmentation algorithms. First, RGB-based image segmentation and the previous method [6] are compared with our results to show importance of the modified spectral imaging system. Then, we compare the segmentation results with the RGBbased k-means [8] and the RGB-based normalized cut algorithms [9]. Experimental results from a number of raw PCBs have shown the effectiveness of the developed method for classification of complicated images.

\section{Spectral Imaging System}

Figure 1 shows the newly constructed spectral imaging system for raw PCBs. The camera system consists of a monochromatic CCD camera (Retiga 1300) with 12-bit dynamic range and Peltier cooling, a macro lens of C-mount connected directly to the camera, VariSpec ${ }^{\mathrm{TM}}$ Liquid Crystal Tunable Filter (LCTF), and a personal computer.

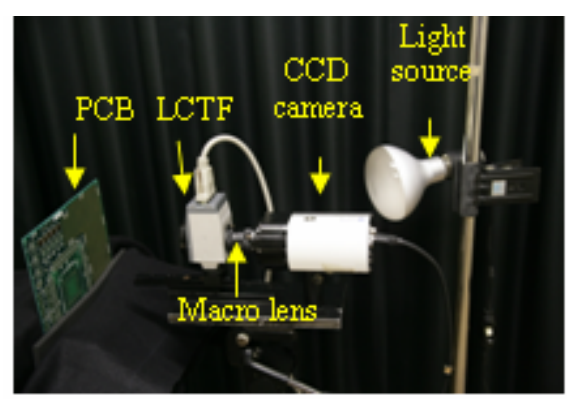

Fig. 1. Imaging system

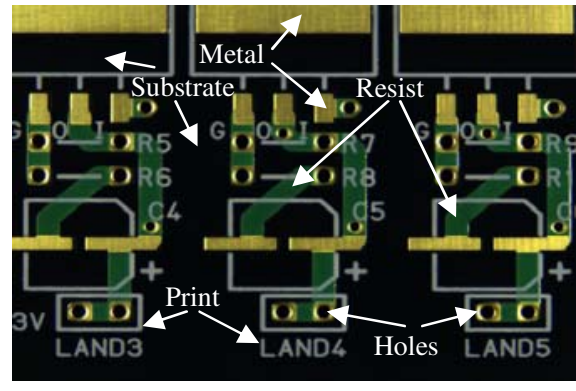

Fig. 2. Partial image of a raw circuit board

We used multiple light sources of incandescent lamps for effective surface illumination. The LCTF has the spectral properties of bandwidth $10 \mathrm{~nm}$ and wavelength 
range [400-720nm]. The image resolution is $1280 \mathrm{x} 1024$ pixels for the area of $35 \mathrm{~mm} \mathrm{x}$ $30 \mathrm{~mm}$. The previous system in [6] had the limited spectral resolution and range of $40 \mathrm{~nm}$ and $[450-650 \mathrm{~nm}]$. Moreover the image resolution and sensitivity are much improved. The viewing direction of the camera is always perpendicular to the board surface as shown in Fig. 1. Figure 2 shows the observed image of a small part on a raw circuit board. The main elements on the circuit board surface are four materials (metal, resist-coated metal, silk-screen print, and substrate) and metal holes.

Figure 3 shows the measuring geometry with multiple light sources. The observed surface reflectance depend not only on the material composition, but also on the surface geometry and roughness. In order to avoid large fluctuation of pixel values between highlight area and matte area, we control the illumination direction of a light source. In our system, we use three incandescent light sources of 300W. Two light sources illuminate the same surface alternatively from one of two directions (from left or right) that are mirrored about the viewing direction. The third light source works as back illumination for detecting holes. We investigated a proper illumination angle for observing PCB materials. We found that the minimum illumination angle is $20^{\circ}$ this is because of the camera shadow on the board. Then the incidence angel $25^{\circ}$ was chosen in our imaging system. Decreasing the incident angle to less than $25^{\circ}$ makes strong specular highlight on the board especially on metal parts, and increasing this angle to more than $25^{\circ}$ makes metal parts more noisy and difficult to classify.

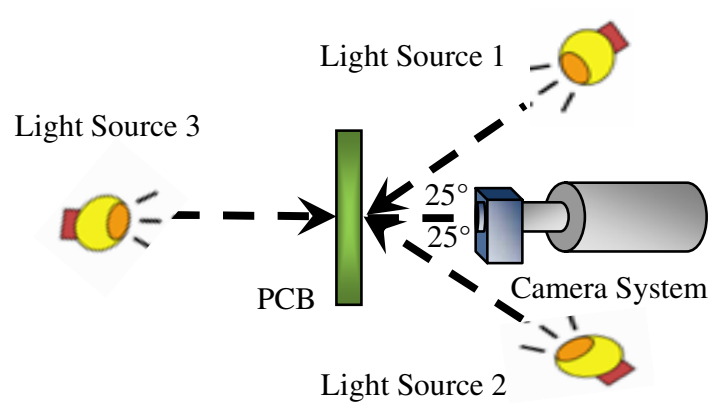

Fig. 3. Measuring geometry with multiple light sources

\section{Reflectance Estimation Based on Material Features}

\subsection{Material Properties}

The PCBs materials reflection properties depend on the measuring geometries. We can divide the PCB materials into two categories of metal parts and non-metal (dielectric) parts on the basis of reflection. In the case of metal, incident light is specularly reflected. Sharp edges of metal flakes and holes produce specular highlights and shadowing effects on the other side. Moreover, for metal surface and footprint material edges at some angles of viewing and lighting strong specular highlights appear. Thus specular reflection and shadowing effects can be controlled by changing direction of light. For dielectric parts, materials surface are smooth and strong specular highlights cannot occur at some illumination directions. According to the dichromatic 
reflection model [10], the diffuse spectral reflectance of these materials is constant. Thus, changing the illumination angle will not have a great effect on the spectral reflectance estimation for such type of materials. The elements of substrate, print, footprint, and resist are classified into this type.

\subsection{Spectral Reflectance Data}

We use a straightforward way of obtaining a reliable estimation of the reflectance function from the camera outputs of narrow band filtration. Let the wavelength bands of the filter be 31 bands of $\lambda_{1}, \lambda_{2}, \ldots, \lambda_{31}$ corresponding to $400,410, \ldots, 700 \mathrm{~nm}$. Let $S\left(\lambda_{k} ; x, y\right)$ be the surface-spectral reflectance at wavelength $\lambda_{k}(k=1,2, \ldots, 31)$ at location of $(x, y)$, which can be recovered by eliminating the illumination effect from the sensor outputs as follows:

$$
S\left(\lambda_{k} ; x, y\right)=\frac{\rho_{k}(x, y)}{\int_{400}^{700} E(\lambda) R_{k}(\lambda) d \lambda},
$$

where $E(\lambda)$ is the illuminant spectral power distribution of the light source, and $R_{k}(\lambda)$ is the $k$-th sensor spectral sensitivity function. This process is repeated for the spectral images from both lightning directions of left and right.

\subsection{Unified Spectral Reflectance}

The light sources illuminate the same surface alternatively from one of two directions. We combine the spectral reflectance data to produce only one spectral reflectance image from two captured images. Because the shape of spectral reflectance characterizes the material properties of each pixel point on the PCB, some features of the spectral curves are used in the combination operation. The proposed combination process is composed of the following steps,

1. Let $\bar{S}_{k}=\overline{S\left(\lambda_{k}, x, y\right)}$ be the average of the observed reflectance at a particular wavelength $\mathrm{k}$ over the entire image region. Then we calculate the average spectral reflectance $\left(\bar{S}_{1}, \bar{S}_{2}, \ldots, \bar{S}_{31}\right)$ from both images.

2. If both pixel values from left and right images are high and the both reflectances achieve the condition $S\left(\lambda_{k} ; x, y\right)>\bar{S}_{k}(k=1,2, \ldots, 31)$, this pixel is classified into the silk-screen print area, and the higher reflectance is chosen.

3. If one pixel value from both images is very high and the other is extremely low, this pixel includes specular highlight of metal. The higher reflectance is chosen for a metal surface. Then we can neglect shadow area on the board.

4. If both pixel values do not have big difference in reflectance, this pixel is classified into dielectric. The average reflectance is calculated.

5. For the remaining pixels except for the material areas extracted in the above, the higher reflectance from both sides is chosen. 


\section{Material Classification and Image Segmentation}

A material classification algorithm is proposed based on the spectral features among the spectral reflectances. The image segmentation process is divided into two sub-processes of pixel-based classification and region growing.

\subsection{Pixel-Based Classification Algorithm}

The following algorithm is applied to each pixel independently. Adjacent pixels with close reflectance are gathered in the same region and used as initial segments for the post processing level.

1. The average spectral reflectance $\left(\bar{S}_{1}, \bar{S}_{2}, \ldots, \bar{S}_{31}\right)$ is calculated for the whole image.

2. Pixels with high reflectance values and satisfy $S\left(\lambda_{k} ; x, y\right)>\bar{S}_{k}(k=1,2, \ldots, 31)$ for the whole visible range [400-700nm] are classified into silk-screen print.

3. The peak wavelength of each spectral curve is detected for the remaining pixels except for screen-print. If the spectral peak exists in the range [600-700nm] and the spectral reflectance values satisfy conditions of $S\left(\lambda_{k} ; x, y\right)>\bar{S}_{k}(k=21,22$, $\ldots, 31)$ and $S\left(\lambda_{k} ; x, y\right)<\bar{S}_{k}(k=1,2, \ldots, 11)$, the pixel is classified into metal.

4. If the peak wavelength in the remaining pixels is in the range [510-590nm] and the relevant spectral reflectance satisfies the conditions $S\left(\lambda_{k} ; x, y\right)>\bar{S}_{k}(k=12$, $13, \ldots, 20)$ and $S\left(\lambda_{k} ; x, y\right)<\bar{S}_{k}(k=21,22, \ldots, 31)$, then the pixel is classified into resist-coated metal.

5. The other pixels satisfying the condition $S\left(\lambda_{k} ; x, y\right)<S_{k}(k=1,2, \ldots, 31)$ are classified into substrate.

6. Finally, the through holes are determined independently from the observed image by using back-illumination. The back-illuminated image is binarized using a threshold, in which the brighter parts correspond to the holes.

\subsection{Region Growing Algorithm}

The above algorithm partitions the spectral image of a PCB into different material regions. However, there are pixels remaining without any labels in the above. In addition, isolated regions with a small number of pixels can be considered as noisy pixels. Hence, an algorithm of merging those undetermined pixels into the neighboring regions is needed. The initial segments are provided by the above pixel-based algorithm. Let us consider the following condition of region homogeneity $R_{j}$

$$
H\left(R_{j}\right)=\text { True }, j=1,2, \ldots, N,
$$

where $N$ is the number of initial segments. The merging process depends on calculating distances between segments $S$ and $S^{\prime}$. We define the distance as a spectral difference in the 31-dimensional vector, which is calculated using Euclidian distance

$$
D=\sqrt{\sum_{i=1}^{K}\left(S_{i}-S_{i}\right)^{2}},
$$


where $K$ is the number of wavelengths. The region growing algorithm is started using a region of $3 \times 3$ pixels with overlapped widows. The minimum distance is checked between the current pixel and surrounding pixels to update the segments. This process continues until the merging of all adjacent regions stop. Finally, a smoothing operation is executed to get the final image segmentation result.

\section{Experiments}

\subsection{Performance of the Proposed Method}

The scene of the raw circuit board shown in Fig. 2 was captured with the present spectral imaging system under incandescent lamps. The image size was $1280 \times 1024$ pixels. Two data sets of surface-spectral reflectances were estimated from the two spectral images at two different light sources. We combined these reflectance images into one reflectance image by comparing the corresponding reflectances at the same pixel point and applying the above rules to all pixels. Then, the proposed classification algorithm was executed for the spectral reflectance image. The typical spectral reflectances obtained for the PCB in Fig. 2 is shown in Fig. 4(a). Figure 4(b) shows the classification results of the developed method.

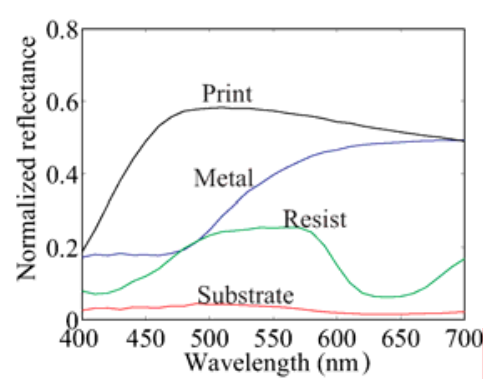

(a)

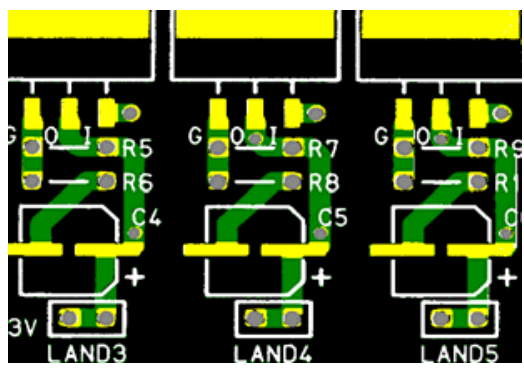

(b)

Fig. 4. (a) Typical curves of surface-spectral reflectance for print, metal, metal, and substrate of the PCB shown in Fig. 2. (b) Material classification results for a part of the raw PCB.

In the figure, the regions classified are painted in different colors, such as white for silk-screen, yellow for metal, green for resist-coated metal, black for substrate and grey for hole. It should be note that the observed PCB image is clearly classified into four material regions and through-holes.

\subsection{Comparison with RGB Reflectance-Based Method}

In order to examine the effectiveness of surface reflectance in material classification, the spectral camera system was replaced with a digital still camera. We used a Canon camera, EOS-1Ds MarkII to capture color images of the same PCB under the same illumination environment. A Kenko extension ring was inserted between the camera 
body and the lens to get the required focus from enough distance. The RGB images with the same size $1280 \times 1024$ as the spectral images were obtained. The normalized color values were calculated as spectral reflectance from Eq. (1) for only R, G, and B channels by eliminating illumination effect. Figure 5 shows the captured RGB image. Figure 6 shows the typical color reflectances obtained for different PCB materials. The classification process based on the RGB reflectances developed as follows:

1. Average color reflectances $\bar{R}, \bar{G}, \bar{B}$ over the whole image are calculated from red, green and blue values.

2. High reflectance pixels satisfying three conditions $R(x, y)>\bar{R}, G(x, y)>\bar{G}$, $B(x, y)>\bar{B}$ are classified into silk-screen print.

3. If the remaining pixels except for the screen print satisfy the conditions $R(x, y)>\bar{R}$ and $R(x, y)>G(x, y)>B(x, y)$, then the pixels are classified into metal.

4. If the remaining pixels satisfy $R(x, y)<B(x, y), B(x, y)<G(x, y)$, then the pixels are classified into resist metal.

5. The other pixels are classified into substrate.

6. Finally, the through holes can be determined by using back-illumination.

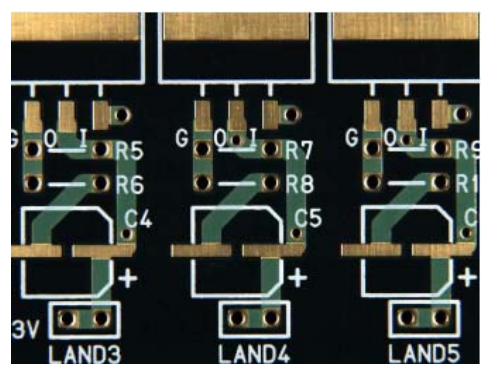

Fig. 5. Captured color image

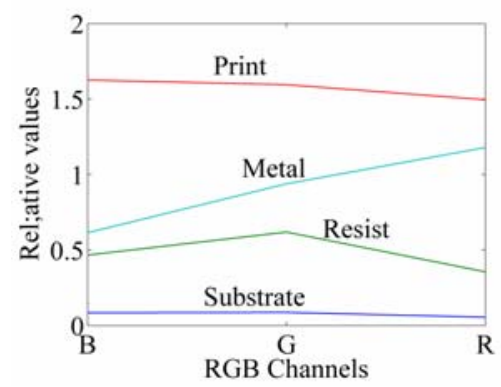

Fig. 6. Materials typical RGB reflectances

Figure 7(d) presents the RGB-based segmentation results using the above classification algorithm without holes detection to easily compare segmentation results. Comparing with Fig. 4(b), we can confirm the accuracy of the proposed reflectancebased classification algorithm. This is clear from the shape of materials, especially metal flakes and metal holes, where the RGB-based algorithm has a lot of missclassified pixels and some other pixels has wrong classification, especially in metal parts with specular highlight area.

\subsection{Segmentation Comparison with K-Means and Normalized Cut Algorithms}

For comparison with a traditional clustering algorithm and a popular graph theoretic algorithm, we choose the k-means [8] and the normalized cut [9] algorithms. Those algorithms require expensive computational cost and memory requirements for large size images. Moreover, the high dimension of the spectral images makes it difficult 


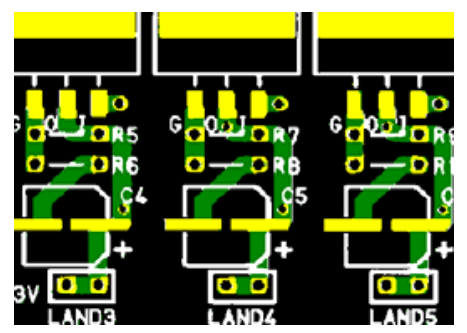

(a) Ground truth

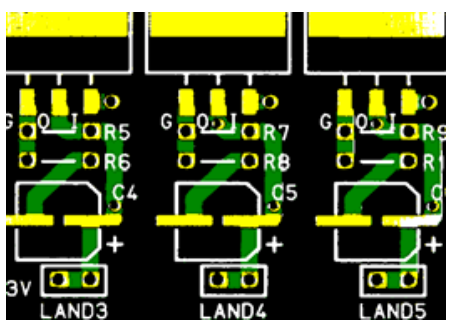

(c) Previous method [6]

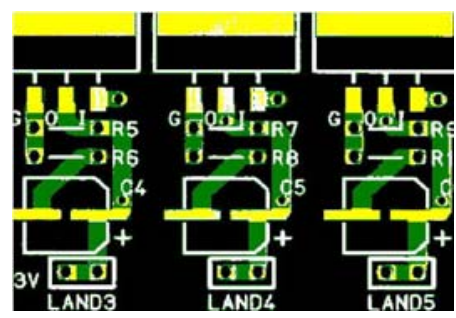

(e) RGB-based K-means

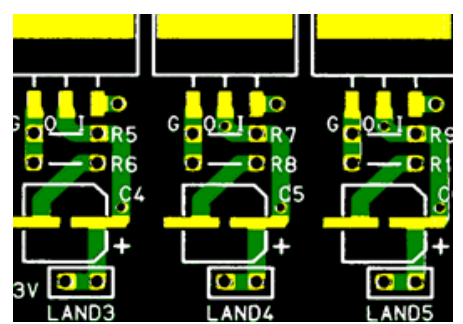

(b) Proposed spectral-based method

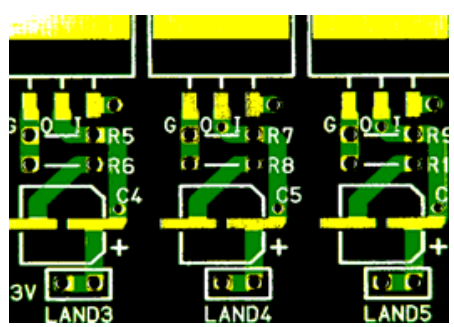

(d) RGB reflectance-based

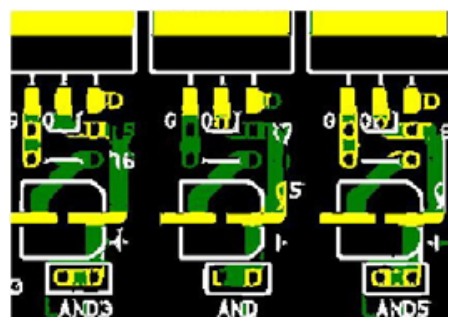

(f) RGB-based N-cut

Fig. 7. Segmentation results by the different methods, compared with the ground truth

to apply such algorithms to the present problem. Therefore, we apply the k-means algorithm to the RGB reflectance image and the normalized cut algorithm to the resized RGB reflectance image to check the performance of our classification method. The final segmentation results for all algorithms are summarized in Fig. 7 without holes detection to easily present the performance of each algorithm in PCB segmentation. Fig. 7(a) shows the ground truth of segmentation. The ground truth is manually generated as a desired segmentation. Fig. 7(b) shows the image segmentation results by the proposed method. Figs. 7 (c)-(f) show the segmentation results by the previous method proposed in [6], RGB reflectance-based method, the k-means clustering, and the normalized cut algorithm, respectively. We changed the initial seed points for kmeans many times but we got nearly same result.

Table 1 lists the accuracy and CPU time of the compared algorithms. The methods are run on CPU Intel Xeon E5405 2GHz with 3G memory. The proposed, previous and RGB methods used C language on FreeBSD software. K-means and N-cut used Matlab on the same system. 
Table 1. Comparison of the accuracy and CPU time for the compared methods

\begin{tabular}{|c|c|c|c|c|c|}
\hline Method & $\begin{array}{c}\text { Proposed } \\
\text { method }\end{array}$ & $\begin{array}{c}\text { Previous } \\
\text { method [6] }\end{array}$ & RGB-based & $\begin{array}{c}\text { RGB-based } \\
\text { K-means }\end{array}$ & $\begin{array}{c}\text { RGB-based } \\
\text { N-cut }\end{array}$ \\
\hline Quality rate & $98.72 \%$ & $96.45 \%$ & $94.01 \%$ & $77.56 \%$ & $74.37 \%$ \\
\hline CPU time (s) & 8.71 & 8.22 & 6.64 & 3.86 & 1321.57 \\
\hline
\end{tabular}

To demonstrate the accuracy of our method, we apply the proposed algorithm on a more complicated four materials PCB. Figure 8 shows segmentation result of a different board with four materials.

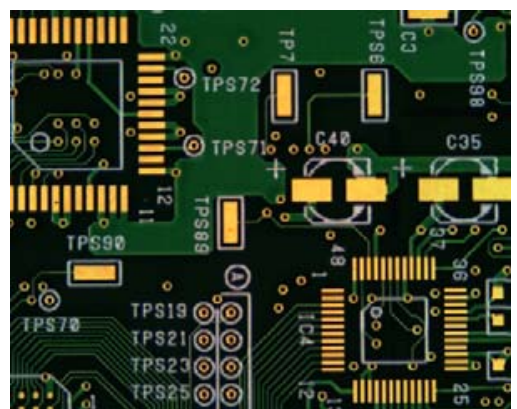

(a) Four materials PCB spectral image

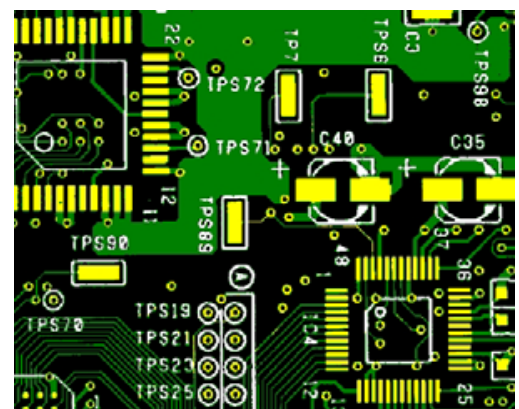

(b) Relevant segmentation result

Fig. 8. Segmentation results of a four material PCB

In case of five materials PCB with footprint elements, the proposed algorithm can easily be extended by calculating the average reflectance for the remaining pixels except print, resist, and metal after step 4 in section 4.1 . Then check step 5 for substrate and the remaining pixels will be footprint. The classification of a five material PCB is presented in Fig 9. We can easily note that the developed method can be used

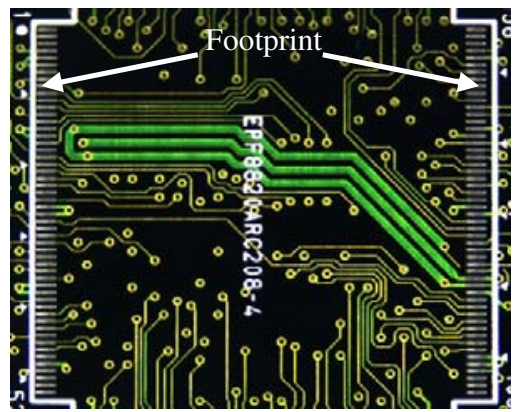

(a) Five materials PCB spectral image

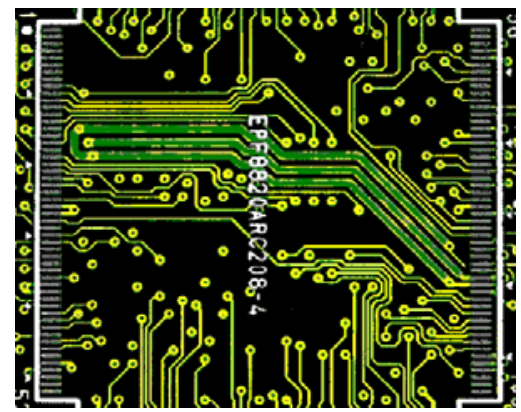

(b) Relevant segmentation result

Fig. 9. Segmentation results of a five material PCB 
for different PCBs with different number of materials. The classification results have shown a high accuracy with CPU time less than 9s for 1280x1024x31 spectral color PCB image.

\section{Conclusion}

This paper has presented an approach to a reliable material classification for PCBs by constructing a spectral imaging system. The system worked in the whole spectral range of visible wavelength [400-700nm] and the high spectral resolution of narrow filtration. An algorithm was presented for effectively classifying the surface material on each pixel into several elements such as substrate, metal, resist, footprint, and paint, based on the surface-spectral reflectance information estimated from the imaging system. The proposed approach was an incorporation of spectral reflectance estimation, spectral feature extraction, and image segmentation processes for material classification of raw PCBs. The performance of the proposed method was compared with the other methods using the previous method, the RGB-reflectance based algorithm, the k-means algorithm and the normalized cut algorithm. The experimental results showed the goodness of the present method in classification accuracy and computational cost. The algorithm can be applied directly to the material classification problem in a variety of raw PCBs.

\section{References}

1. Chang, P.C., Chen, L.Y., Fan, C.Y.: A case-based evolutionary model for defect classification of printed circuit board images. J. Intell. Manuf. 19, 203-214 (2008)

2. Tsai, D.M., Yang, R.H.: An eigenvalue-based similarity measure and its application in defect detection: Image and Vision Computing 23(12), 1094-1101 (2005)

3. Ibrahim, Z., Al-Attas, S.A.R.: Wavelet-based printed circuit board inspection algorithm. Integrated Computer-Aided Engineering 12, 201-213 (2005)

4. Huang, S.Y., Mao, C.W., Cheng, K.S.: Contour-Based Window Extraction Algorithm for Bare Printed Circuit Board Inspection. IEICE Trans. 88-D, 2802-2810 (2005)

5. Leta, F.R., Feliciano, F.F., Martins, F.P.R.: Computer Vision System for Printed Circuit Board Inspection. In: ABCM Symp. Series in Mechatronics, vol. 3, pp. 623-632 (2008)

6. Tominaga, S.: Material Identification via Multi-Spectral Imaging and Its Application to Circuit Boards. In: 10th Color Imaging Conference, Color Science, Systems and Applications, Scottsdale, Arizona, pp. 217-222 (2002)

7. Tominaga, S., Okamoto, S.: Reflectance-Based Material Classification for Printed Circuit Boards. In: 12th Int. Conf. on Image Analysis and Processing, Italy, pp. 238-243 (2003)

8. Duda, R.O., Hart, P.E., Stork, D.G.: Pattern Classification. John Wiley and Sons, New York (2001)

9. Shi, J., Malik, J.: Normalized Cuts and Image Segmentation. IEEE Trans. on Pattern Analysis and Machine Intelligence 22(8), 888-905 (2000)

10. Tominaga, S.: Surface Identification using the Dichromatic Reflection Model. IEEE Trans. PAMI 13, 658-670 (1991) 